\title{
Recursions for the flag-excedance number in colored permutations groups
}

\author{
ELI BAGNO \\ The Jerusalem College of Technology \\ 93721 Jerusalem, Israel \\ email: bagnoe@jct.ac.il \\ and \\ DAVID GARBER \\ Department of Applied Mathematics \\ Holon Institute of Technology \\ 58102 Holon, Israel \\ email: garber@hit.ac.il \\ and \\ TOUFIK MANSOUR \\ Department of Mathematics \\ University of Haifa \\ 31905 Haifa, Israel \\ email: toufik@math.haifa.ac.il \\ and \\ ROBERT SHWARTZ \\ Department of Computer Sciences and Mathematics \\ Ariel University \\ 40700 Ariel, Israel \\ email: robertsh@ariel.ac.il
}

(Received: November 15, 2014, and in revised form May 27, 2015)

\begin{abstract}
The excedance number for $S_{n}$ is known to have an Eulerian distribution. Nevertheless, the classical proof uses descents rather than excedances. We present a direct proof based on a recursion which uses only excedances and extend it to the flag-excedance parameter defined on the group of colored permutations $G_{r, n}=\mathbb{Z}_{r} \prec S_{n}$. We have also computed the distribution of a variant of the flag-excedance number, and show that its enumeration uses the Stirling number of the second kind. Moreover, we show that the generating function of the flag-excedance number defined on $\mathbb{Z}_{r} \succ S_{n}$ is symmetric, and its variant is log-concave on $\mathbb{Z}_{r} \succ S_{n}$..
\end{abstract}

Mathematics Subject Classification(2010). 05A19, 20B30, 05E15.

Keywords: colored permutation group, flag-excedance number, Stirling number of second kind, permutation statistics, recursion.

DOI: $10.1515 /$ puma-2015-0005 


\section{Introduction}

Let $S_{n}$ be the symmetric group on $n$ letters. The parameter excedance, which is defined on a permutation $\pi \in S_{n}$ by:

$$
\operatorname{exc}(\pi)=|\{i \in\{1,2, \ldots, n\} \mid \pi(i)>i\}|,
$$

is well-known (see [16, Vol. I, pp. 135, 186; Vol. II, p. viii], [17]). Another classical parameter defined on permutations of $S_{n}$ is the descent number, defined by:

$$
\operatorname{des}(\pi)=|\{i \in\{1,2, \ldots, n-1\} \mid \pi(i)>\pi(i+1)\}| .
$$

Both parameters have the same distribution, which can be read from the following recursion:

$$
a(n, k)=(k+1) a(n-1, k)+(n-k) a(n-1, k-1),
$$

with the following initial conditions:

$$
a(n, 0)=1, a(0, k)=0 \forall k \forall n,
$$

where $a(n, k)$ is the number of permutations in $S_{n}$ with $k$ excedances or $k$ descents. The generating function $\sum_{\pi \in S_{n}} q^{\operatorname{exc}(\pi)+1}$ is called the Eulerian polynomial.

There is a well-known proof for this recursion by enumerating the descents [14], and there is a bijection from $S_{n}$ onto itself, taking the descents into the excedances [19]. Later, a different proof of this recursion, which uses only excedances, was given independently by Jansonn [15] and by the authors (in this paper).

There are several different definitions of the excedance number for generalizations of the symmetric group. Brenti [8] defined a version for the hyperoctahedral group $B_{n}=\mathbb{Z}_{2} 2 S_{n}$. Chen, Tang and Zhao [9] used this definition to construct a type-B analogue of the derangement polynomials, having properties such as the Sturm sequence property and their coefficients having the spiral property.

A different generalization of the excedance number for the colored permutation groups $G_{r, n}=\mathbb{Z}_{r} 2 S_{n}$ was introduced by Steingrimsson [20]. This version of the excedance number equidistributes with his version of the descent number for the colored permutation groups. He supplies some Eulerian-type recursions for these parameters and presents some geometric applications.

In [5], the first two authors defined a different version of the excedance number for the colored permutation groups, called the flag-excedance number. This definition was motivated by the view of $\mathbb{Z}_{r} 2 S_{n}$ as a subgroup of $\operatorname{Sym}\left(\Sigma_{n}\right)$, where $\Sigma_{n}=\left\{i^{[c]} \mid 1 \leq i \leq n, 0 \leq c<r\right\}$ is the set of $n$ digits colored by $r$ colors:

$$
\operatorname{fexc}(\pi)=\left|\left\{i \in \Sigma_{n} \mid \pi(i)>i\right\}\right| .
$$

One can compute the flag-excedance number in a different way (all the notations will be defined later):

$$
\operatorname{fexc}(\pi)=r \cdot \operatorname{exc}_{A}(\pi)+\operatorname{csum}(\pi) .
$$

A similar approach was used in the definition of the flag major index in the colored permutation groups, see [1, 3, 11]. 
An interesting application of the parameter fexc $(\pi)$ was introduced by Athanasiadis: Consider an $(n-1)$-dimensional simplicial complex $\Delta$ and let $f_{i}(\Delta)$ be the number of $i$-dimensional faces of $\Delta$. The $h$-polynomial of $\Delta$ is defined as:

$$
h(\delta, x)=\sum_{i=0}^{n} f_{i-1}(\Delta) x^{i}(1-x)^{n-i} .
$$

Now, let $V$ be an $n$-element set and let $\Gamma$ be a finite geometric subdivision of the abstract simplicial complex $2^{V}$. The local h-polynomial $\ell_{V}(\Gamma, x)$ is:

$$
\ell_{V}(\Gamma, x)=\sum_{F \subset 2^{V}}(-1)^{n-|F|} h\left(\Gamma_{F}, x\right)
$$

where $\Gamma_{F}$ is the restriction of $\Gamma$ to the face $F \in 2^{V}$ and $h(\Delta, x)$ is the $h$-polynomial of the simplicial complex $\Delta$. The $r$-th edgewise subdivision of a simplicial complex is a standard way to subdivide the complex $\Delta$ in such a way that each face $F \in \Delta$ is subdivided into $r^{\operatorname{dim}(F)}$ faces of the same dimension.

Denote by $\operatorname{sd}(\Delta)^{r}$ the $r$-th barycentric subdivision of $\Delta$. A permutation $\pi \in \mathbb{Z}_{r} \nmid S_{n}$ is called balanced if the parameter $\operatorname{csum}(\pi)$ (which is defined to be the sum of the colors of the digits of $\pi$ ) is a multiple of $r$. The subset of $\mathbb{Z}_{r} \prec S_{n}$, consisting of all the permutations without absolute fixed points, is defined as:

$$
D_{n}^{r}=\left\{\pi \in \mathbb{Z}_{r} \prec S_{n} \mid \forall i \in\{1,2, \ldots, n\}, \pi(i) \neq i\right\} .
$$

Let $\left(D_{n}^{r}\right)^{b}$ denote the set of all balanced permutations in $D_{n}^{r}$. Then, Athansiadis [2] shows the following result:

TheOREM 1.1 (Athanasiadis) Let $V$ be an n-element set. Then

$$
\ell_{V}\left(\operatorname{sd}\left(2^{V}\right)^{r}, q\right)=\sum_{\pi \in\left(D_{n}^{r}\right)^{b}} q^{\frac{\operatorname{fexc}(\pi)}{r}} .
$$

We survey here some other results dealing with the flag-excedance parameter defined on $G_{r, n}$.

In [5], the multi-distributions of the excedance number with some natural parameters were computed. In [4], these definitions and results were generalized to the so-called multi-colored permutation group $\left(\mathbb{Z}_{r_{1}} \times \cdots \times \mathbb{Z}_{r_{k}}\right)$ 乙 $S_{n}$. In [6], the multi-distribution of the excedance number with the number of fixed points on the set of involutions in $G_{r, n}$ was computed. In [18], Mansour and Sun consider similar problems in more general cases.

Recently, Foata and Han [12, 13, have found that this version of the excedance number is equidistributed with some version of the descent number for generalized permutation groups. Moreover, Clark and Ehrenborg [10] mentioned this version of the excedance number as a possible candidate for a generalization for an excedance statistic for all finite Coxeter groups.

We start this paper by presenting a classical way to obtain recursion (1) using only counting of excedances. This argument appears also in [15].

By our definition of excedance, we generalize this recursion for the cases of the hyperoctahedral group $B_{n}=G_{2, n}$ and the colored permutation groups $G_{r, n}$ (all the notations will be defined in the sequel): 
Proposition 1.2 Define:

$$
f_{i}^{A}(r, n, k)=\left|\left\{\pi \in G_{r, n} \mid \operatorname{exc}_{A}(\pi)=k, \operatorname{csum}(\pi)=i\right\}\right| .
$$

Then:

$$
\begin{aligned}
f_{i}^{A}(r, n, k)= & (n-k) f_{i}^{A}(r, n-1, k-1)+(k+1) f_{i}^{A}(r, n-1, k)+ \\
& +\sum_{j=1}^{r-1}\left[(n-k) f_{i-j}^{A}(r, n-1, k)+(k+1) f_{i-j}^{A}(r, n-1, k+1)\right],
\end{aligned}
$$

with the following initial conditions:

$$
f_{i}^{A}(r, n, 0)=\sum_{\substack{\left(t_{1}, \ldots, t_{j}\right), j \leq i \\
1 \leq t_{1}<t_{2}<\cdots<t_{j} \leq n}} j !\left(\begin{array}{c}
i-1 \\
i-j
\end{array}\right)(j+1)^{n-t_{j}} \prod_{u=1}^{j} u^{t_{u}-t_{u-1}-1}
$$

where $t_{0}=0$, and

$$
f_{i}^{A}(r, 0, k)=0 ; f_{0}^{A}(r, 1,0)=1 ; f_{-1}^{A}(r, n, k)=0 \forall n \forall k .
$$

We have also computed the distribution of a variant of the flag-excedance number, denoted by $\operatorname{exc}_{A}$. The interesting point is that its enumeration uses the Stirling number of the second kind:

Proposition 1.3 The number of permutations $\pi$ in $G_{r, n}$ which satisfy $\operatorname{exc}_{A}(\pi)=k$ is given by:

$$
r \sum_{j=1}^{n} \sum_{i=0}^{j-1}(-1)^{k+j-1-i} r^{i} j ! S_{n, j}\left(\begin{array}{c}
j-1 \\
i
\end{array}\right)\left(\begin{array}{c}
n-1-i \\
k
\end{array}\right)
$$

where $S_{n, j}$ is the $(n, j)$-Stirling number of the second kind.

It is well-known that the generating function $\sum_{\pi \in S_{n}} q^{\operatorname{exc}(\pi)}=\sum_{i=0}^{d} a_{i} q^{i}$ has some symmetry properties. It is symmetric in the sense that $a_{i}=a_{d-i}$ for $i \in\left\{1, \ldots,\left\lfloor\frac{d}{2}\right\rfloor\right\}$. We prove here the corresponding symmetry property for $G_{r, n}$. We also prove that its variant $\operatorname{exc}_{A}$ is $\log$-concave in $G_{r, n}$.

The paper is organized as follows. In Section 2, we introduce the colored permutation group $G_{r, n}=\mathbb{Z}_{r} 2 S_{n}$ and we define some of its parameters and statistics. Section 3 deals with the proof of the recursion for $S_{n}$. In Sections 4 and 5 , we give the corresponding recursions for $B_{n}$ and $G_{r, n}$, respectively. Section 6 deals with the distribution of the parameter $\operatorname{exc}_{A}$, which involves the Stirling number of the second kind. In Section 7 , we present the symmetry of the generating function of the excedance number, and in Section 8 we prove the log-concavity property of the parameter $\operatorname{exc}_{A}$.

Acknowledgements: We would like to thank an anonymous referee for his advices. 


\section{The group of colored permutations and its statistics}

DEFINITION 2.1 Let $r$ and $n$ be positive integers. The group of colored permutations of $n$ digits with $r$ colors is the wreath product

$$
G_{r, n}=\mathbb{Z}_{r} 2 S_{n}=\mathbb{Z}_{r}^{n} \rtimes S_{n}
$$

consisting of all pairs $(\vec{z}, \tau)$, where $\vec{z}$ is an $n$-tuple of integers between 0 and $r-1$ and $\tau \in S_{n}$. The multiplication is defined by the following rule: for $\vec{z}=\left(z_{1}, \ldots, z_{n}\right)$ and $\overrightarrow{z^{\prime}}=\left(z_{1}^{\prime}, \ldots, z_{n}^{\prime}\right)$,

$$
(\vec{z}, \tau) \cdot\left(\overrightarrow{z^{\prime}}, \tau^{\prime}\right)=\left(\left(z_{1}+z_{\tau^{-1}(1)}^{\prime}, \ldots, z_{n}+z_{\tau^{-1}(n)}^{\prime}\right), \tau \circ \tau^{\prime}\right)
$$

(the operation + is taken modulo $r$ ).

Here is an example for the multiplication in $G_{5,3}$ :

$$
\left((2,1,0),\left(\begin{array}{lll}
1 & 2 & 3 \\
2 & 1 & 3
\end{array}\right)\right) \cdot\left((2,2,0),\left(\begin{array}{lll}
1 & 2 & 3 \\
2 & 3 & 1
\end{array}\right)\right)=\left((4,3,0),\left(\begin{array}{lll}
1 & 2 & 3 \\
1 & 3 & 2
\end{array}\right)\right) .
$$

Another way to present $G_{r, n}$ is as follows: Consider the alphabet

$$
\Sigma=\left\{1, \ldots, n, \overline{1}, \ldots, \bar{n}, \ldots, 1^{[r-1]}, \ldots, n^{[r-1]}\right\}
$$

as the set $\{1, \ldots, n\}$ colored by the colors $0, \ldots, r-1$. Then, an element of $G_{r, n}$ is a colored permutation, i.e., a bijection $\pi: \Sigma \rightarrow \Sigma$ satisfying the following condition: if $\pi\left(i^{[\alpha]}\right)=j^{[\beta]}$, then $\pi\left(i^{[\alpha+1]}\right)=$ $j^{[\beta+1]}$ (the addition in the exponents is taken modulo $r$ ). Using this approach, the element $\pi=$ $\left(\left(z_{1}, \ldots, z_{n}\right), \tau\right) \in G_{r, n}$ is the permutation of $\Sigma$, satisfying $\pi(i)=\pi\left(i^{[0]}\right)=\tau(i)^{\left[z_{\tau(i)}\right]}$ for each $1 \leq i \leq n$. For example, the element $\pi=\left((2,1,0,3,0,0),\left(\begin{array}{cccccc}1 & 2 & 3 & 4 & 5 & 6 \\ 2 & 1 & 4 & 3 & 6 & 5\end{array}\right)\right) \in G_{6,6}$ satisfies:

$$
\pi(1)=2^{[1]}, \pi(2)=1^{[2]}, \pi(3)=4^{[3]}, \pi(4)=3^{[0]}, \pi(5)=6^{[0]}, \pi(6)=5^{[0]} .
$$

For an element $\pi=(\vec{z}, \tau) \in G_{r, n}$ with $\vec{z}=\left(z_{1}, \ldots, z_{n}\right)$, we write $z_{i}(\pi)=z_{i}$, and denote $|\pi|=(\overrightarrow{0}, \tau)$. We define also $c_{i}(\pi)=r-z_{i}\left(\pi^{-1}\right)$ and $\vec{c}(\pi)=\vec{c}=\left(c_{1}, \ldots, c_{n}\right)$. Using this notation, the element $\pi=(\vec{z}, \tau)=\left((2,1,0,3,0,0),\left(\begin{array}{llllll}1 & 2 & 3 & 4 & 5 & 6 \\ 2 & 1 & 4 & 3 & 6 & 5\end{array}\right)\right)$ satisfies $\vec{c}=(1,2,3,0,0,0)$.

We usually write $\pi$ in its window notation (or one line notation): $\pi=\left(a_{1}^{\left[c_{1}\right]} \cdots a_{n}^{\left[c_{n}\right]}\right)$, where $a_{i}=\tau(i)$, so in the above example, we have: $\pi=\left(2^{[1]} 1^{[2]} 4^{[3]} 3^{[0]} 6^{[0]} 5^{[0]}\right)$ or just $(\overline{2} \overline{\overline{1}} \overline{\overline{4}} 365)$.

Note that $z_{i}$ is the color of the digit $i$ ( $i$ is taken from the window notation), while $c_{j}$ is the color of the digit $\tau(j)$. Here, $j$ stands for the place, whence $i$ stands for the value.

In particular, $G_{1, n}=\mathbb{Z}_{1} \prec S_{n}$ is the classical symmetric group $S_{n}$, while $G_{2, n}=\mathbb{Z}_{2} \prec S_{n}$ is the group of signed permutations $B_{n}$, also known as the hyperoctahedral group, or the classical Coxeter group of type $B$.

Given any ordered alphabet $\Sigma^{\prime}$, we recall the definition of the excedance set of a permutation $\pi$ on $\Sigma^{\prime}($ see [5]):

$$
\operatorname{Exc}(\pi)=\left\{i \in \Sigma^{\prime} \mid \pi(i)>i\right\}
$$

and the flag-excedance number is defined to be $\operatorname{fexc}(\pi)=|\operatorname{Exc}(\pi)|$. 
Definition 2.2 The color order on $\Sigma$ is defined to be:

$$
1^{[r-1]}<\cdots<n^{[r-1]}<1^{[r-2]}<2^{[r-2]}<\cdots<n^{[r-2]}<\cdots<1<\cdots<n .
$$

EXAMPLE 2.3 Given the color order:

$$
\overline{\overline{1}}<\overline{\overline{2}}<\overline{\overline{3}}<\overline{1}<\overline{2}<\overline{3}<1<2<3,
$$

we write $\sigma=(3 \overline{1} \overline{\overline{2}}) \in G_{3,3}$ in an extended form:

$$
\left(\begin{array}{lll|lll|lll}
\overline{\overline{1}} & \overline{\overline{2}} & \overline{\overline{3}} & \overline{1} & \overline{2} & \overline{3} & 1 & 2 & 3 \\
\overline{\overline{3}} & 1 & \overline{2} & \overline{3} & \overline{\overline{1}} & 2 & 3 & \overline{1} & \overline{\overline{2}}
\end{array}\right)
$$

and compute: $\operatorname{Exc}(\sigma)=\{\overline{\overline{1}}, \overline{\overline{2}}, \overline{\overline{3}}, \overline{1}, \overline{3}, 1\}$ and $\operatorname{fexc}(\sigma)=6$.

We present now an alternative way to compute the flag-excedance number. Let $\sigma \in G_{r, n}$. We define:

$$
\operatorname{csum}(\sigma)=\sum_{i=1}^{n} c_{i}(\sigma) .
$$

Note that in the case $r=2$ (i.e. the group $B_{n}$ ), the alphabet $\Sigma$ can be seen as containing the digits $\{ \pm 1, \ldots, \pm n\}$ and the parameter $\operatorname{csum}(\pi)$ counts the number of digits $i \in[n]$ such that $\pi(i)<0$, so it is also called $\operatorname{neg}(\pi)$.

Define now:

$$
\operatorname{Exc}_{A}(\sigma)=\{i \in\{1,2, \ldots, n-1\} \mid \sigma(i)>i\}
$$

where the comparison is with respect to the color order, and denote:

$$
\operatorname{exc}_{A}(\sigma)=\left|\operatorname{Exc}_{A}(\sigma)\right| \text {. }
$$

EXAmple 2.4 Given $\sigma=(\overline{1} \overline{\overline{3}} 4 \overline{2}) \in G_{3,4}$, we have $\operatorname{csum}(\sigma)=4, \operatorname{Exc}_{\mathrm{A}}(\sigma)=\{3\}$ and hence $\operatorname{exc}_{A}(\sigma)=1$.

We have now (see [5]):

LEMMA 2.5

$$
\operatorname{fexc}(\sigma)=r \cdot \operatorname{exc}_{A}(\sigma)+\operatorname{csum}(\sigma)
$$

A similar result for the flag major index statistic was achieved by Adin and Roichman [1].

\section{The recursion for $S_{n}$}

We supply a classical proof for the recursion for the Eulerian polynomial using its interpretation as a generating function for the excedance number for $S_{n}$ (this proof appears independently in [15]). Denote by $a(n, k)$ the number of permutations in $S_{n}$ with exactly $k$ excedances. Then we have the following recursion: 
Proposition 3.1

$$
a(n, k)=(k+1) a(n-1, k)+(n-k) a(n-1, k-1),
$$

with the following initial conditions:

$$
a(n, 0)=1, a(0, k)=0, \forall n \forall k .
$$

Proof. For any $n$ and $0 \leq k \leq n-1$, denote by $S(n, k)$ the set of permutations in $S_{n}$ with exactly $k$ excedances. Denote also:

$$
R=\left\{\pi \in S(n, k) \mid \pi^{-1}(n)<\pi(n)\right\}
$$

and

$$
T=\left\{\pi \in S(n, k) \mid \pi^{-1}(n) \geq \pi(n)\right\} .
$$

Define $\Phi: S(n, k) \rightarrow S(n-1, k) \cup S(n-1, k-1)$ as follows: Let $\pi \in S(n, k)$. Then $\Phi(\pi)$ is the permutation in $S_{n-1}$ obtained from $(n, \pi(n)) \pi$ by ignoring the last digit.

Let $\pi \in S(n, k)=R \cup T$. If $\pi \in R$, then $\Phi(\pi) \in S(n-1, k)$. Note that $\left|\Phi^{-1}(\Phi(\pi))\right|=k+1$. On the other hand, if $\pi \in T$, then $\Phi(\pi) \in S(n-1, k-1)$ and $\left|\Phi^{-1}(\Phi(\pi))\right|=n-1-(k-1)=n-k$.

We give the following example for illustrating the proof.

Example 3.2 Consider $S(5,2)$, i.e. the set of permutations in $S_{5}$ having exactly 2 excedances.

Let

$$
R \ni \pi=\left(\begin{array}{ccccc}
(1) & (2) & 3 & 4 & 5 \\
5 & 3 & 1 & 2 & 4
\end{array}\right) \mapsto\left(\begin{array}{ccccc}
(1) & (2) & 3 & 4 & 5 \\
\mathbf{4} & 3 & 1 & 2 & \mathbf{5}
\end{array}\right) \mapsto\left(\begin{array}{cccc}
(1) & (2) & 3 & 4 \\
4 & 3 & 1 & 2
\end{array}\right)=\Phi(\pi),
$$

so:

$$
\Phi^{-1}(\Phi(\pi))=\left\{\left(\begin{array}{ccccc}
(1) & (2) & 3 & 4 & 5 \\
5 & 3 & 1 & 2 & 4
\end{array}\right),\left(\begin{array}{ccccc}
(1) & (2) & 3 & 4 & 5 \\
4 & 5 & 1 & 2 & 3
\end{array}\right),\left(\begin{array}{ccccc}
(1) & (2) & 3 & 4 & 5 \\
4 & 3 & 1 & 2 & 5
\end{array}\right)\right\}
$$

Let

$$
T \ni \pi=\left(\begin{array}{ccccc}
1 & 2 & (3) & 4 & 5 \\
5 & 2 & 4 & 3 & 1
\end{array}\right) \mapsto\left(\begin{array}{ccccc}
1 & 2 & (3 & 4 & 5 \\
\mathbf{1} & 2 & 4 & 3 & 5
\end{array}\right) \mapsto\left(\begin{array}{cccc}
1 & 2 & (3) & 4 \\
1 & 2 & 4 & 3
\end{array}\right)=\Phi(\pi) .
$$

Then:

$$
\Phi^{-1}(\Phi(\pi))=\left\{\left(\begin{array}{ccccc}
(1) & 2 & (3) & 4 & 5 \\
5 & 2 & 4 & 3 & 1
\end{array}\right),\left(\begin{array}{ccccc}
1 & (2) & (3) & 4 & 5 \\
1 & 5 & 4 & 3 & 2
\end{array}\right),\left(\begin{array}{ccccc}
1 & 2 & (3) & (4) & 5 \\
1 & 2 & 4 & 5 & 3
\end{array}\right)\right\} .
$$

\section{The recursion for $B_{n}$}

In this section, we generalize the above recursion to $B_{n}=\mathbb{Z}_{2}$ ? $S_{n}$. We start with some notation.

$$
\begin{aligned}
F_{i}^{A}(n, k)=\left\{\pi \in B_{n} \mid \operatorname{exc}_{A}(\pi)=k, \operatorname{neg}(\pi)=i\right\} \\
\\
f_{i}^{A}(n, k)=\left|F_{i}^{A}(n, k)\right|, \\
F_{i}(n, k)=\left\{\pi \in B_{n} \mid \operatorname{fexc}(\pi)=k, \operatorname{neg}(\pi)=i\right\}
\end{aligned}
$$




$$
\begin{gathered}
f_{i}(n, k)=\left|F_{i}(n, k)\right|, \\
F(n, k)=\bigcup_{i=0}^{n} F_{i}(n, k) \\
f(n, k)=\sum_{i=0}^{n} f_{i}(n, k) .
\end{gathered}
$$

In $B_{n}$, we have $\operatorname{fexc}(\pi)=2 \cdot \operatorname{exc}_{A}(\pi)+\operatorname{neg}(\pi)$, hence:

$$
f_{i}(n, k)=f_{i}^{A}\left(n, \frac{k-i}{2}\right) .
$$

In the following proposition, we give a recursion for $f_{i}^{A}(n, k)$ :

Proposition 4.1

$$
\begin{aligned}
f_{i}^{A}(n, k)= & (n-k) f_{i}^{A}(n-1, k-1)+(k+1) f_{i}^{A}(n-1, k)+ \\
& +(n-k) f_{i-1}^{A}(n-1, k)+(k+1) f_{i-1}^{A}(n-1, k+1),
\end{aligned}
$$

with the following initial conditions:

$$
f_{i}^{A}(n, 0)=\sum_{\substack{\left(t_{1}, \ldots, t_{i}\right) \\ 1 \leq t_{1}<t_{2}<\cdots<t_{i} \leq n}} i !(i+1)^{n-t_{i}} \prod_{u=1}^{i} u^{t_{u}-t_{u-1}-1},
$$

where $0 \leq i \leq n$ and $t_{0}=0$, and

$$
f_{i}^{A}(0, k)=0 ; f_{0}^{A}(1,0)=1 ; f_{-1}^{A}(n, k)=0 \forall n \forall k .
$$

Proof. We start with the proof of the recursion. Define:

$$
\begin{gathered}
F_{i, 0}^{A}(n, k)=\left\{\pi \in B_{n} \mid \operatorname{exc}_{A}(\pi)=k, \operatorname{neg}(\pi)=i, \pi^{-1}(n)>0\right\} \\
f_{i, 0}^{A}(n, k)=\left|F_{i, 0}^{A}(n, k)\right| \\
F_{i, 1}^{A}(n, k)=\left\{\pi \in B_{n} \mid \operatorname{exc}_{A}(\pi)=k, \operatorname{neg}(\pi)=i, \pi^{-1}(n)<0\right\} \\
f_{i, 1}^{A}(n, k)=\left|F_{i, 1}^{A}(n, k)\right|
\end{gathered}
$$

Obviously, $F_{i}^{A}(n, k)=F_{i, 0}^{A}(n, k) \cup F_{i, 1}^{A}(n, k)$, and hence:

$$
f_{i}^{A}(n, k)=f_{i, 0}^{A}(n, k)+f_{i, 1}^{A}(n, k) .
$$

Define $\Phi_{0}: F_{i, 0}^{A}(n, k) \rightarrow F_{i}^{A}(n-1, k) \cup F_{i}^{A}(n-1, k-1)$ as follows: Let $\pi \in F_{i, 0}^{A}(n, k)$. Then $\Phi_{0}(\pi)$ is the permutation of $B_{n-1}$ obtained from $(n, \pi(n)) \pi$ by ignoring the last digit. 
Now, define:

$$
R_{0}=\left\{\pi \in F_{i, 0}^{A}(n, k) \mid \pi^{-1}(n)<\pi(n)\right\}
$$

and

$$
T_{0}=\left\{\pi \in F_{i, 0}^{A}(n, k) \mid \pi^{-1}(n) \geq \pi(n)\right\} .
$$

Let $\pi \in F_{i, 0}^{A}(n, k)=R_{0} \cup T_{0}$. If $\pi \in R_{0}$, then $\Phi_{0}(\pi) \in F_{i}^{A}(n-1, k)$. Note that $\left|\Phi_{0}^{-1}\left(\Phi_{0}(\pi)\right)\right|=k+1$. On the other hand, if $\pi \in T_{0}$, then $\Phi_{0}(\pi) \in F_{i}^{A}(n-1, k-1)$ and $\left|\Phi_{0}^{-1}\left(\Phi_{0}(\pi)\right)\right|=n-1-(k-1)=n-k$.

Define $\Phi_{1}: F_{i, 1}^{A}(n, k) \rightarrow F_{i-1}^{A}(n-1, k) \cup F_{i-1}^{A}(n-1, k+1)$ similarly.

Now, define:

$$
R_{1}=\left\{\pi \in F_{i, 1}^{A}(n, k)|| \pi^{-1}(n) \mid<\pi(n)\right\}
$$

and

$$
T_{1}=\left\{\pi \in F_{i, 1}^{A}(n, k)|| \pi^{-1}(n) \mid \geq \pi(n)\right\} .
$$

Let $\pi \in F_{i, 1}^{A}(n, k)=R_{1} \cup T_{1}$. If $\pi \in R_{1}$, then $\Phi_{1}(\pi) \in F_{i-1}^{A}(n-1, k+1)$. Note that $\left|\Phi_{1}^{-1}\left(\Phi_{1}(\pi)\right)\right|=$ $k+1$. On the other hand, if $\pi \in T_{1}$, then $\Phi_{1}(\pi) \in F_{i-1}^{A}(n-1, k)$ and $\left|\Phi_{1}^{-1}\left(\Phi_{1}(\pi)\right)\right|=n-1-(k-1)=$ $n-k$.

Combining together all the parts, we get the desired recursion for $f_{i}^{A}(n, k)$.

Now we prove the initial condition:

$$
f_{i}^{A}(n, 0)=\sum_{\substack{\left(t_{1}, \ldots, t_{i}\right) \\ 1 \leq t_{1}<t_{2}<\cdots<t_{i} \leq n}} i !(i+1)^{n-t_{i}} \prod_{u=1}^{i} u^{t_{u}-t_{u-1}-1}
$$

where $t_{0}=0$.

Let $\pi \in B_{n}$ and let $1 \leq t_{1}<\cdots<t_{i} \leq n$ be such that $\pi\left(t_{j}\right)<0$ for all $1 \leq j \leq i$.

In order to insure that $\operatorname{exc}_{A}(\pi)=0$, we have to require that for each $\ell \notin\left\{t_{1}, \ldots, t_{i}\right\}, \pi(\ell) \leq \ell$. For each $1 \leq \ell<t_{1}$ (if there are any), we have only one possibility: $\pi(\ell)=\ell$. For $t_{1}<\ell<t_{2}$ (if there are any), we have exactly two possibilities, and so on: for $t_{m}<\ell<t_{m+1}, 1 \leq m \leq i-1$ (if there are any), we have exactly $m+1$ possibilities. Finally, for $t_{i}<\ell \leq n$, we have exactly $i+1$ possibilities.

After fixing $\pi(\ell)$ for each $\ell \notin\left\{t_{1}, \ldots, t_{i}\right\}$, we have exactly $i$ ! possibilities for locating $\pi\left(t_{j}\right)$, for $1 \leq j \leq i$. This gives us the desired initial condition.

The following example should clarify the proof for the initial condition. Let $\pi \in B_{9}$ and assume that $t_{1}=3, t_{2}=6, t_{3}=8$. Then in order to get $\operatorname{exc}_{A}(\pi)=0$, we must have $\pi(1)=1, \pi(2)=2$. $\pi(4)$ can be 3 or 4 . $\pi(5) \in\{3,4,5\}$ but once $\pi(4)$ has been chosen we have only 2 possibilities for it. $\pi(7) \in\{3,4,5,6,7\}$ which yields 3 possibilities and for $\pi(9)$ we have 4 possibilities. The values corresponding to $\{\pi(3), \pi(6), \pi(8)\}$ are already fixed, so we just have to order them.

\section{The corresponding recursion for $G_{r, n}$}

The recursion for $B_{n}$ can be generalized to $G_{r, n}=\mathbb{Z}_{r} \prec S_{n}$ very easily. We continue with similar notations.

$$
F_{i}^{A}(r, n, k)=\left\{\pi \in G_{r, n} \mid \operatorname{exc}_{A}(\pi)=k, \operatorname{csum}(\pi)=i\right\}
$$




$$
\begin{gathered}
f_{i}^{A}(r, n, k)=\left|F_{i}^{A}(r, n, k)\right|, \\
F_{i}(r, n, k)=\left\{\pi \in G_{r, n} \mid \operatorname{fexc}(\pi)=k, \operatorname{csum}(\pi)=i\right\} \\
f_{i}(r, n, k)=\left|F_{i}(r, n, k)\right|, \\
F(r, n, k)=\bigcup_{i=0}^{(r-1) n} F_{i}(r, n, k) \\
f(r, n, k)=\sum_{i=0}^{(r-1) n} f_{i}(r, n, k) .
\end{gathered}
$$

In $G_{r, n}$, we have $\operatorname{fexc}(\pi)=r \cdot \operatorname{exc}_{A}(\pi)+\operatorname{csum}(\pi)$, hence:

$$
f_{i}(r, n, k)=f_{i}^{A}\left(r, n, \frac{k-i}{r}\right) .
$$

In the following proposition, we give a recurrence for $f_{i}^{A}(r, n, k)$ :

Proposition 5.1

$$
\begin{aligned}
f_{i}^{A}(r, n, k)= & (n-k) f_{i}^{A}(r, n-1, k-1)+(k+1) f_{i}^{A}(r, n-1, k)+ \\
& +\sum_{j=1}^{r-1}\left[(n-k) f_{i-j}^{A}(r, n-1, k)+(k+1) f_{i-j}^{A}(r, n-1, k+1)\right],
\end{aligned}
$$

with the following initial conditions:

$$
f_{i}^{A}(r, n, 0)=\sum_{\substack{\left(t_{1}, \ldots, t_{j}\right), j \leq i \\
1 \leq t_{1}<t_{2}<\cdots<t_{j} \leq n}} j !\left(\begin{array}{c}
i-1 \\
i-j
\end{array}\right)(j+1)^{n-t_{j}} \prod_{u=1}^{j} u^{t_{u}-t_{u-1}-1},
$$

where $0 \leq i \leq n$ and $t_{0}=0$, and

$$
f_{i}^{A}(r, 0, k)=0 ; f_{0}^{A}(r, 1,0)=1 ; f_{-1}^{A}(r, n, k)=0 \forall n \forall k .
$$

For completeness, we present here the proof for the general case.

Proof. We start with the proof of the recursion. Define for all $j$ such that $0 \leq j \leq r-1$ :

$$
\begin{gathered}
F_{i, j}^{A}(r, n, k)=\left\{\pi \in G_{r, n} \mid \operatorname{exc}_{A}(\pi)=k, \operatorname{csum}(\pi)=i, c_{n}(\pi)=j\right\} \\
f_{i, j}^{A}(r, n, k)=\left|F_{i, j}^{A}(r, n, k)\right|
\end{gathered}
$$

Obviously, $F_{i}^{A}(r, n, k)=\bigcup_{j=0}^{r-1} F_{i, j}^{A}(r, n, k)$, and hence:

$$
f_{i}^{A}(r, n, k)=\sum_{j=0}^{r-1} f_{i, j}^{A}(r, n, k) .
$$


Define $\Phi_{0}: F_{i, 0}^{A}(r, n, k) \rightarrow F_{i}^{A}(r, n-1, k) \cup F_{i}^{A}(r, n-1, k-1)$ as follows: Let $\pi \in F_{i, 0}^{A}(r, n, k)$. Then $\Phi_{0}(\pi)$ is the permutation of $G_{r, n-1}$ obtained from $(n, \pi(n)) \pi$ by ignoring the last digit.

Now, define:

$$
R_{0}=\left\{\pi \in F_{i, 0}^{A}(r, n, k) \mid \pi^{-1}(n)<\pi(n)\right\}
$$

and

$$
T_{0}=\left\{\pi \in F_{i, 0}^{A}(r, n, k) \mid \pi^{-1}(n) \geq \pi(n)\right\} .
$$

Let $\pi \in F_{i, 0}^{A}(r, n, k)=R_{0} \cup T_{0}$. If $\pi \in R_{0}$, then $\Phi_{0}(\pi) \in F_{i}^{A}(r, n-1, k)$. Note that $\left|\Phi_{0}^{-1}\left(\Phi_{0}(\pi)\right)\right|=$ $k+1$. On the other hand, if $\pi \in T_{0}$, then $\Phi_{0}(\pi) \in F_{i}^{A}(r, n-1, k-1)$ and $\left|\Phi_{0}^{-1}\left(\Phi_{0}(\pi)\right)\right|=n-1-(k-1)=$ $n-k$.

For all $j$ such that $1 \leq j \leq r-1$, define:

$$
\Phi_{j}: F_{i, j}^{A}(r, n, k) \rightarrow F_{i-j}^{A}(r, n-1, k) \cup F_{i-j}^{A}(r, n-1, k+1)
$$

similarly.

Now, define:

$$
R_{j}=\left\{\pi \in F_{i, j}^{A}(r, n, k)|| \pi^{-1}(n) \mid<\pi(n)\right\}
$$

and

$$
T_{j}=\left\{\pi \in F_{i, j}^{A}(r, n, k)|| \pi^{-1}(n) \mid \geq \pi(n)\right\} .
$$

Let $\pi \in F_{i, j}^{A}(r, n, k)=R_{j} \cup T_{j}$. If $\pi \in R_{j}$, then $\Phi_{j}(\pi) \in F_{i-j}^{A}(r, n-1, k+1)$. Note that $\left|\Phi_{j}^{-1}\left(\Phi_{j}(\pi)\right)\right|=k+1$. On the other hand, if $\pi \in T_{j}$, then $\Phi_{j}(\pi) \in F_{i-j}^{A}(r, n-1, k)$ and $\left|\Phi_{j}^{-1}\left(\Phi_{j}(\pi)\right)\right|=$ $n-1-(k-1)=n-k$.

Combining together all the parts, we get the desired recursion for $f_{i}^{A}(r, n, k)$.

Now we prove the initial condition:

$$
f_{i}^{A}(r, n, 0)=\sum_{\substack{\left(t_{1}, \ldots, t_{j}\right), j \leq i \\
1 \leq t_{1}<t_{2}<\cdots<t_{j} \leq n}} j !\left(\begin{array}{c}
i-1 \\
i-j
\end{array}\right)(j+1)^{n-t_{j}} \prod_{u=1}^{j} u^{t_{u}-t_{u-1}-1}
$$

where $t_{0}=0$.

Let $\pi \in G_{r, n}$. Let $j \leq i$ and let $\left(t_{1}, \cdots, t_{j}\right)$ be such that $1 \leq t_{1}<\cdots<t_{j} \leq n$ and $c_{t_{k}}(\pi)>0$ (for $1 \leq k \leq j)$

In order to insure that $\operatorname{exc}_{A}(\pi)=0$, we require that for each $\ell \notin\left\{t_{1}, \ldots, t_{j}\right\}, \pi(\ell) \leq \ell$. For each $1 \leq \ell<t_{1}$ (if there are any), we have only one possibility: $\pi(\ell)=\ell$. For $t_{1}<\ell<t_{2}$ (if there are any), we have exactly two possibilities, and so on: for $t_{m}<\ell<t_{m+1}, 1 \leq m \leq j-1$ (if there are any), we have exactly $m+1$ possibilities. Finally, for $t_{j}<\ell \leq n$, we have exactly $j+1$ possibilities.

After fixing $\pi(\ell)$ for each $\ell \notin\left\{t_{1}, \ldots, t_{j}\right\}$, we have exactly $j !\left(\begin{array}{c}i-1 \\ i-j\end{array}\right)$ possibilities to locate $\pi\left(t_{k}\right)$, $1 \leq k \leq j$, and to color them in such a way that $\operatorname{csum}(\pi)=i$. This gives us the desired initial condition for $G_{r, n}$. 


\section{The distribution of the parameter $\operatorname{exc}_{A}$}

Recall that:

$$
f_{i}^{A}(r, n, k)=\left|\left\{\pi \in G_{r, n} \mid \operatorname{exc}_{A}(\pi)=k, \operatorname{csum}(\pi)=i\right\}\right| .
$$

Let $d(r, n, k)=\sum_{i=0}^{(r-1) n} f_{i}^{A}(r, n, k)$ be the number of permutations $\pi \in G_{r, n}$ having $\operatorname{exc}_{A}(\pi)=k$.

Proposition 5.1 gives that:

$$
\begin{aligned}
d(r, n, k)= & (n-k) d(r, n-1, k-1)+(k+1) d(r, n-1, k) \\
& +(n-k)(r-1) d(r, n-1, k)+(k+1)(r-1) d(r, n-1, k+1),
\end{aligned}
$$

which is equivalent to:

$$
\begin{aligned}
d(r, n, k)= & (n-k) d(r, n-1, k-1) \\
& +[(k+1)+(r-1)(n-k)] d(r, n-1, k) \\
& +(k+1)(r-1) d(r, n-1, k+1) .
\end{aligned}
$$

In order to solve this recurrence, we define the following generating polynomial:

$$
D_{r, n}(t)=\sum_{k=0}^{n} d(r, n, k) t^{k}
$$

Rewriting Equation (3) in terms of the polynomial $D_{r, n}(t)$ yields:

$$
\begin{aligned}
D_{r, n}(t)= & n t D_{r, n-1}(t)-t \frac{\partial}{\partial t}\left(t D_{r, n-1}(t)\right) \\
& +(1+(r-1) n) D_{r, n-1}(t)-(r-2) t \frac{\partial}{\partial t} D_{r, n-1}(t) \\
& +(r-1) \frac{\partial}{\partial t} D_{r, n-1}(t),
\end{aligned}
$$

which implies that:

$$
D_{r, n}(t)=[r n+(n-1)(t-1)] D_{r, n-1}(t)-(t-1)(t+r-1) \frac{\partial}{\partial t} D_{r, n-1}(t) .
$$

Now, in order to simplify this recurrence, assume that $D_{r, n}(t)$ can be written as $D_{r, n}(t)=P_{r, n}(t) E_{r, n}(t)$. We will give later the condition which $P_{r, n}(t)$ has to satisfy. Therefore, Equation (4) can be written in terms of $P_{r, n}(t)$ and $E_{r, n}(t)$ as

$$
\begin{aligned}
E_{r, n}(t)= & \frac{[r n+(n-1)(t-1)] P_{r, n-1}(t)-(t-1)(t+r-1) \frac{\partial}{\partial t} P_{r, n-1}(t)}{P_{r, n}(t)} E_{r, n-1}(t) \\
& -\frac{(t-1)(t+r-1) P_{r, n-1}(t)}{P_{r, n}(t)} \frac{\partial}{\partial t} E_{r, n-1}(t) .
\end{aligned}
$$


Let us assume that

$$
[r n+(n-1)(t-1)] P_{r, n-1}(t)=(t-1)(t+r-1) \frac{\partial}{\partial t} P_{r, n-1}(t) .
$$

One solution of the above differential equation is:

$$
P_{r, n}(t)=\frac{(t-1)^{n+1}}{t+r-1} .
$$

Note that $\frac{P_{r, n-1}(t)}{P_{r, n}(t)}=\frac{1}{t-1}$. Therefore, for all $n \geq 1$,

$$
E_{r, n}(t)=-(t+r-1) \frac{\partial}{\partial t} E_{r, n-1}(t) .
$$

Checking the recurrence for $n=1$, we have by a direct computation that:

$$
E_{r, 1}(t)=\frac{D_{r, 1}(t)}{P_{r, 1}(t)}=\frac{r}{\left(\frac{(t-1)^{2}}{t+r-1}\right)}=-(t+r-1) \frac{\partial}{\partial t} E_{r, 0}(t),
$$

which gives that:

$$
\frac{\partial}{\partial t} E_{r, 0}(t)=-\frac{r}{(t-1)^{2}}
$$

Hence, we define $E_{r, 0}(t)=\frac{r}{t-1}$.

Proposition 6.1 For all $n \geq 1$,

$$
E_{r, n}(t)=(-1)^{n-1} r \sum_{j=1}^{n} j ! S_{n, j} \frac{(t+r-1)^{j}}{(1-t)^{j+1}}
$$

where $S_{n, j}$ is the $(n, j)$-Stirling number of the second kind.

Proof. By induction on $n$, the recurrence relation (5) gives that:

$$
E_{r, n}(t)=(-1)^{n} \sum_{j=1}^{n} S_{n, j}(t+r-1)^{j} \frac{\partial^{j}}{\partial t^{j}} E_{r, 0}(t) .
$$

Using the initial condition of this recurrence, namely $E_{r, 0}(t)=\frac{r}{t-1}$, we obtain that:

$$
E_{r, n}(t)=(-1)^{n} \sum_{j=1}^{n} S_{n, j}(t+r-1)^{j} \frac{(-1)^{j} j ! r}{(t-1)^{j+1}} .
$$

This is equivalent to:

$$
E_{r, n}(t)=(-1)^{n-1} r \sum_{j=1}^{n} j ! S_{n, j} \frac{(t+r-1)^{j}}{(1-t)^{j+1}}
$$

which completes the proof.

Now we are ready to give an explicit formula for the polynomial $D_{r, n}(t)$. 
Corollary 6.2 For all $n \geq 1$,

$$
D_{r, n}(t)=r \sum_{j=1}^{n} j ! S_{n, j}(t+r-1)^{j-1}(1-t)^{n-j} .
$$

Proof. From the definitions, we have that:

$$
D_{r, n}(t)=P_{r, n}(t) E_{r, n}(t)=\frac{(t-1)^{n+1}}{t+r-1} E_{r, n}(t)
$$

Hence, by Proposition 6.1, we get the desired result.

Finding the coefficient of $t^{k}$ in the polynomial $D_{r, n}(t)$, we obtain an explicit formula for $d(r, n, k)$, as follows:

Theorem 6.3 The number of permutations $\pi \in G_{r, n}$ which satisfy $\operatorname{exc}_{A}(\pi)=k$ is given by:

$$
d(r, n, k)=r \sum_{j=1}^{n} \sum_{i=0}^{j-1}(-1)^{k+j-1-i} r^{i} j ! S_{n, j}\left(\begin{array}{c}
j-1 \\
i
\end{array}\right)\left(\begin{array}{c}
n-1-i \\
k
\end{array}\right),
$$

where $S_{n, j}$ is the $(n, j)$-Stirling number of the second kind.

\section{$7 \quad$ Symmetry of the flag-excedance number}

In this section, we present the symmetry property of the flag-excedance number:

THEOREM 7.1 The generating polynomial

$$
\sum_{\pi \in G_{r, n}} q^{\mathrm{fexc}(\pi)}=\sum_{i=0}^{r n-1} a_{i} q^{i}
$$

satisfies: $a_{i}=a_{r n-1-i}$ for $i \in\left\{1, \ldots,\left\lfloor\frac{r n-1}{2}\right\rfloor\right\}$.

Define a bijection of $G_{r, n}: \pi \mapsto \pi^{\prime}$ in the following way: For $1 \leq i \leq n-1$, if $\pi(i)=j^{[\beta]}$, then $\pi^{\prime}(n-i)=(n+1-j)^{[r-\beta]}$ and if $\pi(n)=j^{[\beta]}$, then $\pi^{\prime}(n)=(n+1-j)^{[r-1-\beta]}$.

Instead of burdening the reader with the subtle though standard proof, we choose to give an example of the bijection:

$$
\begin{aligned}
\pi & =\left(\begin{array}{llll|llll|llll}
\overline{\overline{1}} & \overline{\overline{2}} & \overline{\overline{3}} & \overline{\overline{4}} & \overline{1} & \overline{2} & \overline{3} & \overline{4} & 1 & 2 & 3 & 4 \\
2 & \overline{1} & 4 & \overline{\overline{3}} & \overline{\overline{2}} & 1 & \overline{\overline{4}} & \overline{3} & \overline{2} & \overline{\overline{1}} & \overline{4} & 3
\end{array}\right) \\
\pi^{\prime} & =\left(\begin{array}{llll|llll|llll}
\overline{\overline{1}} & \overline{\overline{2}} & \overline{\overline{3}} & \overline{\overline{4}} & \overline{1} & \overline{2} & \overline{3} & \overline{4} & 1 & 2 & 3 & 4 \\
\overline{1} & 4 & \overline{3} & 2 & 1 & \overline{\overline{4}} & 3 & 2 & \overline{\overline{1}} & \overline{4} & \overline{\overline{3}} & \overline{\overline{2}}
\end{array}\right) .
\end{aligned}
$$




\section{Log-concavity of the parameter $\operatorname{exc}_{A}$}

In this section, we show that some variant of the excedance parameter is log-concave. We start by proving that $\operatorname{exc}_{A}$ on $B_{n}$ is log-concave. The corresponding proof for $G_{r, n}$ is similar.

TheOREm 8.1 The parameter $\operatorname{exc}_{A}$ on $B_{n}$ is log-concave.

Proof. Recall from Section 4 the following definitions:

$$
\begin{gathered}
F_{i}^{A}(n, k)=\left\{\pi \in B_{n} \mid \operatorname{exc}_{A}(\pi)=k, \operatorname{neg}(\pi)=i\right\}, \\
f_{i}^{A}(n, k)=\left|F_{i}^{A}(n, k)\right| .
\end{gathered}
$$

Define:

$$
X_{n, k}=\sum_{i=0}^{n} f_{i}^{A}(n, k) .
$$

Note that $X_{n, k}$ is the number of permutations $\pi \in B_{n}$ having $\operatorname{exc}_{A}(\pi)=k$.

By the recursion given in Proposition 4.1.

$$
\begin{aligned}
f_{i}^{A}(n, k)= & (n-k) f_{i}^{A}(n-1, k-1)+(k+1) f_{i}^{A}(n-1, k)+ \\
& +(n-k) f_{i-1}^{A}(n-1, k)+(k+1) f_{i-1}^{A}(n-1, k+1),
\end{aligned}
$$

we have:

$$
\begin{aligned}
X_{n, k} & =(n-k) X_{n-1, k-1}+(k+1) X_{n-1, k}+(n-k) X_{n-1, k}+(k+1) X_{n-1, k+1}= \\
& =(n-k) X_{n-1, k-1}+(n+1) X_{n-1, k}+(k+1) X_{n-1, k+1} .
\end{aligned}
$$

We prove the log-concavity by induction. For $n=3$, the claim can be easily verified. Now we assume it for $n-1$, and we have to show that:

$$
X_{n, k}^{2} \geq X_{n, k-1} X_{n, k+1}
$$

Along the following computation, we abbreviate $X_{n-1, j}$ to $X_{j}$. We compute:

$$
\begin{aligned}
& X_{n, k}^{2}-X_{n, k-1} X_{n, k+1}= {\left[(n-k) X_{k-1}+(n+1) X_{k}+(k+1) X_{k+1}\right]^{2}-} \\
&-\left[(n-k+1) X_{k-2}+(n+1) X_{k-1}+k X_{k}\right] \cdot \\
& \cdot\left[(n-k-1) X_{k}+(n+1) X_{k+1}+(k+2) X_{k+2}\right]= \\
&=\quad\left[(n-k)^{2} X_{k-1}^{2}+(n+1)^{2} X_{k}^{2}+(k+1)^{2} X_{k+1}^{2}+\right. \\
& \quad+2(n-k)(n+1) X_{k-1} X_{k}+2(n-k)(k+1) X_{k-1} X_{k+1}+ \\
&\left.\quad+2(n+1)(k+1) X_{k} X_{k+1}\right]- \\
& \quad\left[(n-k+1)(n-k-1) X_{k-2} X_{k}+(n-k+1)(n+1) X_{k-2} X_{k+1}+\right. \\
& \quad+(n-k+1)(k+2) X_{k-2} X_{k+2}+(n+1)(n-k-1) X_{k-1} X_{k}+ \\
& \quad+(n+1)^{2} X_{k-1} X_{k+1}+(n+1)(k+2) X_{k-1} X_{k+2}+ \\
&\left.\quad+k(n-k-1) X_{k}^{2}+k(n+1) X_{k} X_{k+1}+k(k+2) X_{k} X_{k+2}\right]=
\end{aligned}
$$




$$
\begin{aligned}
= & {\left[(n-k)^{2} X_{k-1}^{2}-(n-k+1)(n-k-1) X_{k-2} X_{k}\right]+} \\
& +\left[(k+1)^{2} X_{k+1}^{2}-k(k+2) X_{k} X_{k+2}\right]+ \\
& +\left[2(n-k)(n+1) X_{k-1} X_{k}-(n-k+1)(n+1) X_{k-2} X_{k+1}-\right. \\
& \left.\quad-(n+1)(n-k-1) X_{k-1} X_{k}\right]+ \\
+ & {\left[2(n+1)(k+1) X_{k} X_{k+1}-(n+1)(k+2) X_{k-1} X_{k+2}-\right.} \\
& \left.\quad-k(n+1) X_{k} X_{k+1}\right]+ \\
+ & {\left[(n+1)^{2} X_{k}^{2}+2(n-k)(k+1) X_{k-1} X_{k+1}-\right.} \\
& \quad-(n-k+1)(k+2) X_{k-2} X_{k+2}-(n+1)^{2} X_{k-1} X_{k+1}- \\
& \left.\quad-k(n-k-1) X_{k}^{2}\right]
\end{aligned}
$$

We treat each one of the five brackets in the last expression separately.

By the induction hypothesis, the first bracket is greater (or equal) than $X_{k-1}^{2}$. Similarly, the second bracket is greater (or equal) than $X_{k+1}^{2}$.

Since $X_{k-2} X_{k+1} \leq X_{k-1} X_{k}$ by the log-concavity assumption, we have:

$$
\begin{gathered}
2(n-k)(n+1) X_{k-1} X_{k}-(n-k+1)(n+1) X_{k-2} X_{k+1}-(n+1)(n-k-1) X_{k-1} X_{k} \geq \\
\geq 2(n-k)(n+1) X_{k-1} X_{k}-(n-k+1)(n+1) X_{k-1} X_{k}-(n+1)(n-k-1) X_{k-1} X_{k}=0 X_{k-1} X_{k}=0,
\end{gathered}
$$

and hence the third bracket is non-negative. Similarly, the fourth bracket is non-negative too.

From the first two brackets, we have two positive elements: $X_{k-1}^{2}$ and $X_{k+1}^{2}$. Their sum can be written as $\left(X_{k-1}-X_{k+1}\right)^{2}+2 X_{k-1} X_{k+1}$. Adding $2 X_{k-1} X_{k+1}$ to the fifth bracket, we have:

$$
\begin{gathered}
(n+1)^{2} X_{k}^{2}+2((n-k)(k+1)+1) X_{k-1} X_{k+1}-(n-k+1)(k+2) X_{k-2} X_{k+2}- \\
-(n+1)^{2} X_{k-1} X_{k+1}-k(n-k-1) X_{k}^{2} .
\end{gathered}
$$

Since $(n+1)^{2}=((n-k)+(k+1))^{2}=(n-k)^{2}+(k+1)^{2}+2(n-k)(k-1)$, we can simplify the bracket into:

$$
\begin{gathered}
(n+1)^{2} X_{k}^{2}-(n-k+1)(k+2) X_{k-2} X_{k+2} \\
-\left((k+1)^{2}-1\right) X_{k-1} X_{k+1}-\left((n-k)^{2}-1\right) X_{k-1} X_{k+1}-k(n-k-1) X_{k}^{2} .
\end{gathered}
$$

By the log-concavity assumption, this sum is greater (or equal) than:

$$
(n+1)^{2} X_{k}^{2}-(n-k+1)(k+2) X_{k}^{2}-\left((k+1)^{2}-1\right) X_{k}^{2}-\left((n-k)^{2}-1\right) X_{k}^{2}-k(n-k-1) X_{k}^{2},
$$

which is equal to 0 . So, we have that the sum of all five brackets is non-negative and hence we are done. 


\section{References}

[1] R. M. Adin And Y. RoIchman, The flag major index and group actions on polynomial rings, European J. Combin., 22 (2001) 431-446.

[2] C. A. Athanasiadis, Edgewise subdivisions, local h-polynomials and excedances in the wreath product $\mathbb{Z}_{r} \prec S_{n}, \quad$ SIAM J. Discrete Math., 28 (2014) 1479-1492.

[3] E. Bagno and R. Biagioli, Colored-descent representations of complex reflection groups $G(r, p, n), \quad$ Israel J. Math., 160 (2007) 317-347.

[4] E. Bagno, A. Butman And D. Garber, Statistics on the multi-colored permutation groups, Electron. J. Combin., 14 (2007) \#R24.

[5] E. Bagno And D. GaRBeR, On the excedance number of colored permutation groups, Sém. Lothar. Combin., B53f (2006) 17 pp.

[6] E. Bagno, D. Garber and T. Mansour, Excedance number for involutions in complex reflection groups, Sém. Lothar. Combin., B56d (2007) 11 pp.

[7] M. Bona, Combinatorics of permutations, Chapman and Hall/CRC, Second Edition, 2012.

[8] F. BREnTI, q-Eulerian polynomials arising from Coxeter groups, European J. Combin., 15 (1994) 417-441.

[9] W. Y. C. Chen, R. L. TAng And A. F. Y. Zhao, Derangement polynomials and excedances of type B, Electron. J. Combin., 16 (2009) \#R15.

[10] E. Clark And R. Ehrenborg, Excedances of affine permutations, Adv. in Appl. Math., 46 (2011) 175-191.

[11] M. FIRE, Statistics on wreath products, (2005) at http://arxiv.org/abs/math/0409421.

[12] D. Foata And G. Han, The decrease value theorem with an application to permutation statistics, Adv. in Appl. Math., 46 (2011) 296-311.

[13] D. Foata and G. Han, The Flag-Descent and-Excedance numbers, (2009) preprint. (Electronic version: http://www-irma.u-strasbg.fr/ foata/paper/pub116b.html)

[14] D. Foata And M.-P. Schützenberger, Théorie géométrique des polynômes eulériens, Lecture Notes in Math., Vol. 138, Springer-Verlag, Berlin-New York, 1970.

[15] F. JAnsson, Variations on the excedance statistic in permutations, (2006) preprint. (Electronic version: http://www.math.chalmers.se/ einar/exjobb/fredrik-jansson.pdf)

[16] P. A. MacMahon, Combinatory Analysis, Vol. 1 and 2, Cambridge Univ. Press, Cambridge, 1915 (reprinted by Chelsea, New York, 1955). 
[17] P. A. Macmahon, The indices of permutations and the derivation therefrom of functions of a single varaible asscoiated with the permutations of any assemblage of objects, Amer. J. Math., 35 (1913) 281-322.

[18] T. Mansour And Y. Sun, Excedance numbers for permutations in complex reflection groups, Sém. Lothar. Combin., B58b (2008) 7 pp.

[19] R. P. Stanley, Enumerative Combinatorics, Volume I, Cambridge University Press, 2002.

[20] E. Steingrímsson, Permutation statistics of indexed permutations, European J. Combin., 15 (1994) 187-205. 